\title{
Subdivisions de la forêt de bois de couleur à l'île de la Réunion
}

\author{
J.L. DUPOUEY et T. CADET * \\ I.N.R.A., Lahoratoire de Phyto-écologie forestière \\ Centre de Recherches forestieres de Nancy, Champenoux, F 54280 Sichamps \\ * Université de la Rétunion \\ Faculté des Sciences, B.P. 15, 97490 Sainte-Clotilde, La Réunion
}

\begin{abstract}
Résumé
Le traitement informatique par analyse factorielle des correspondances des relevés de végétation forestière de l'île de la Réunion publiés par CADET (1980) nous permet de préciser les subdivisions de la forêt réunionnaise. La validité de la coupure entre série mégatherme hygrophile et série mésotherme hygrophile est confirmée. Par contre, il apparaît deux sous-unités à la série mégatherme : la forêt de basse altitude et la forêt de moycnne altitude. Les espèces différenciant chacune de ces unités sont identifiées.
\end{abstract}

Mots clés : Ile de la Réunion, phytosociologie, forêt tropicale, analyse multivariable, protection de la nature.

\section{Introduction}

A l'exception de quelques lambeaux de végétation semi-xérophile, deux grandes unités physionomiques sont à distinguer dans la forêt réunionnaise :

- les forêts complexes dites de «bois de couleur»qui ceinturent l'île, entre 0 et $1900 \mathrm{~m}$ dans la région au vent et entre 750 et $1100 \mathrm{~m}$ dans la région sous le vent :

- la forêt paucispécifique à dominante de tamarin des hauts (Acacia heterophy/la (i)) qui occupe les pentes situćes entre 1100 et $2000 \mathrm{~m}$ dans les régions abritécs de l'alizé et quelques secteurs sur le versant plus humide.

Les premières constituent un ensemble hétérogène sur le plan écologique. Rıvals (1952) les subdivise en trois sous-étages altitudinaux (fig. 1 a) :

- la forêt complexe de basse altitude;

- la forêt complexe de moyenne altitude;

- la forêt très hygrophile de haute altitude.

(1) La nomenclature utilisće est celle de CaDEt (1980). 


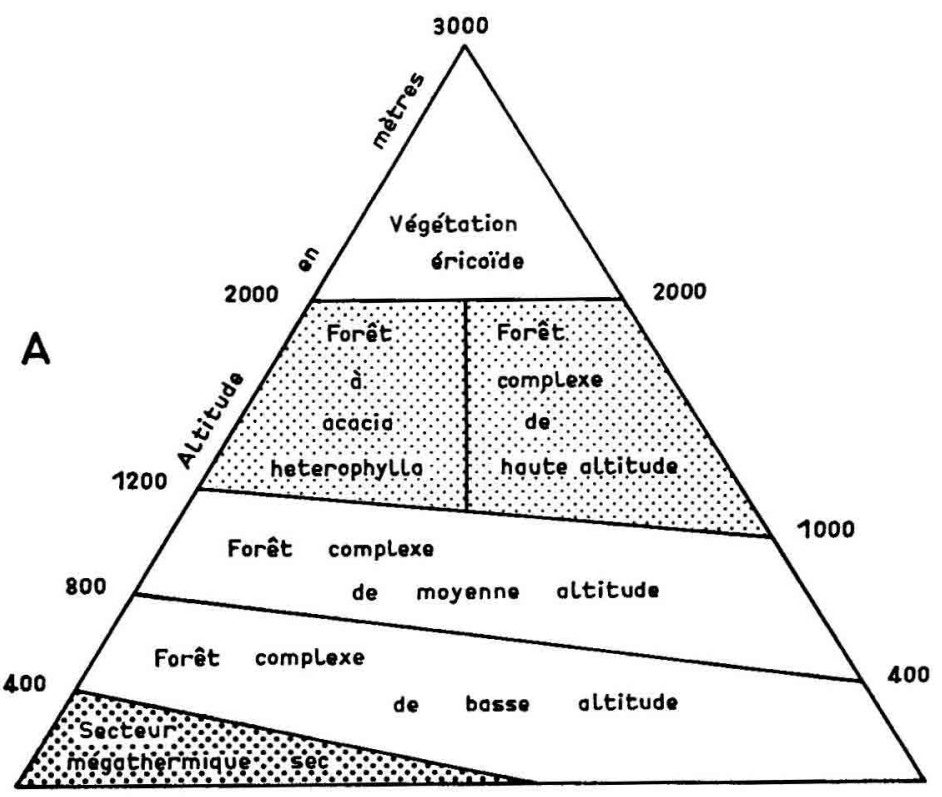

$\mathrm{N}-\mathrm{O}$

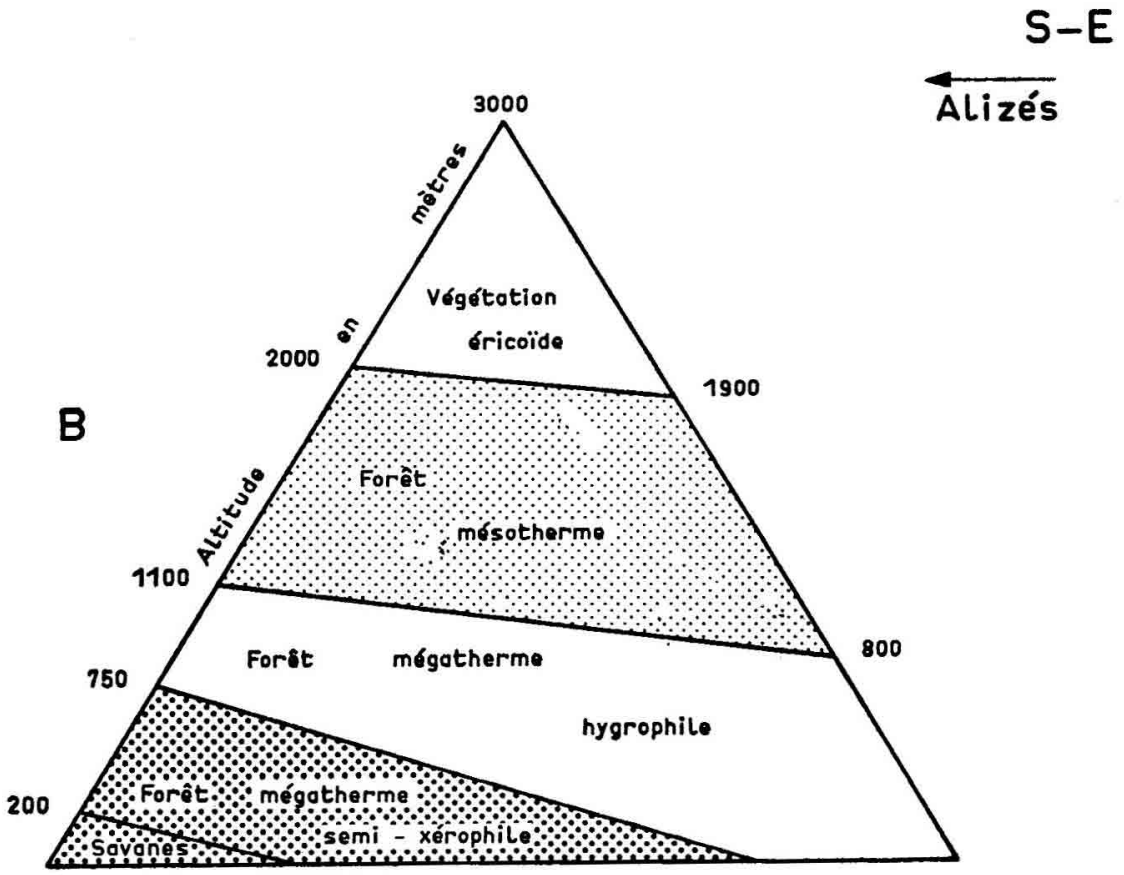

FIG. 1

Etagement de la végétation à la Réunion selon Rivals $(A)$ et Cadet $(B)$.

Altitudinal zonality of vegetution of Reunion island according to Rivals (A) and CADET (B). 
La forêt de tamarins des hauts forme une entité distincte. CADET (1980), pour sa part, ne distingue que deux séries (fig. 1 b) :

- la série mégatherme hygrophile qui regroupe les forêts complexes de basse altitude et unc partie des forêts complexes de moyenne altitude ;

- la série mésotherme hygrophile qui correspond aux forêts très hygrophiles de haute altitude, à une partie des forêts complexes de moyenne altitude et qui inclut la forêt de tamarins des hauts.

Qu'en est-il en fait des subdivisions de ces forêts ? Cette question est primordiale pour l'aménagement forestier. Miguet (1955) exprime ainsi le principe d'action de l'Office National des Forêts pour la zone des bois de couleur : «à la forêt complexe aux mélanges inextricables, nous nous proposons de substituer un peuplement beaucoup plus simple, comportant en principe une à deux essences dominantes seulement ». La flore d'origine est en effet riche de plus de 250 espèces, soit endémiques de la Réunion, soit, pour la plupart, endémiques des Mascareignes. Il s'impose de délimiter des zones de réserve absolue, conservatoires des espèces non cultivées!

La connaissance précise du milieu naturel est donc nécessaire pour choisir les limites des unités de gestion, en particulier celles des surfaces à mettre en réserve, et pour orienter le mode de traitement à appliquer dans les parcelles en culture.

Lanalyse informatique des relevés de végétation forestière collectés par CanET n’avait pas encore été réalisée. Elle nous a permis d’identifier avec objectivité les discontinuités écologiques existantes et les espèces repères s'y rapportant.

\section{Méthode}

Nous avons pris en compte dans lanalyse la totalité des relevés publićs par CaDer (1980) concernant les séries mégatherme hygrophile (cf. CADET, 1980, tabl. VI, p. 129131 ) et mésotherme hygrophile (cf. CADET, 1980, tabl. X, p. 171-172) soit 45 relevés regroupant 272 espèces. Ces relevés ont été collectés par un échantillonnage subjectif des zones forestières non encore dégradées de l'île.

L’analyse factorielle des correspondances est l'outil statistique le mieux adapté à l'analyse des tableaux de relevés. Cette analyse multivariable est maintenant d'utilisation courante dans les études de végétation à la suite des travaux de DaGNisle (1960), Lacoste \& Roux $(1971,1972)$... Rappelons seulement qu'il s'agit d'une méthode de calcul numérique qui permet, après avoir dégagé les directions d'allongement privilégiées du nuage de points étudiés (relevés $\times$ espèces), de projeter ces points sur ces axes principaux ou facteurs pour avoir une approximation du nuage originel dans un espace de dimensions restreintes. On dispose ainsi d'un mode de représentation graphique pour ordoinner simultanément les relevés et les espèces en fonction de leur ressemblance.

Nous lavons appliquée aux tableaux floristiques complets (espèces accidentelles incluses) en ne prenant en compte que la seule présence-absence des espèces. 


\section{Résultats}

\subsection{Analyse globale}

La figure 2 montre la répartition des relevés dans le plan des axes 1 et 2 de lanalyse factorielle globale. On constate la séparation très nette entre relevés de la forêt mégatherme, groupés à l'extrémité positive de l'axe 1, et relevé de la forêt mésotherme aux abcisses négatives. Ce premier axe participe d'ailleurs pour 22 p. 100 à la variance totale de l'ensemble des relevés et a done un haut degré de signification. Ainsi le bien fondé de la coupure entre les deux séries est confirmé.

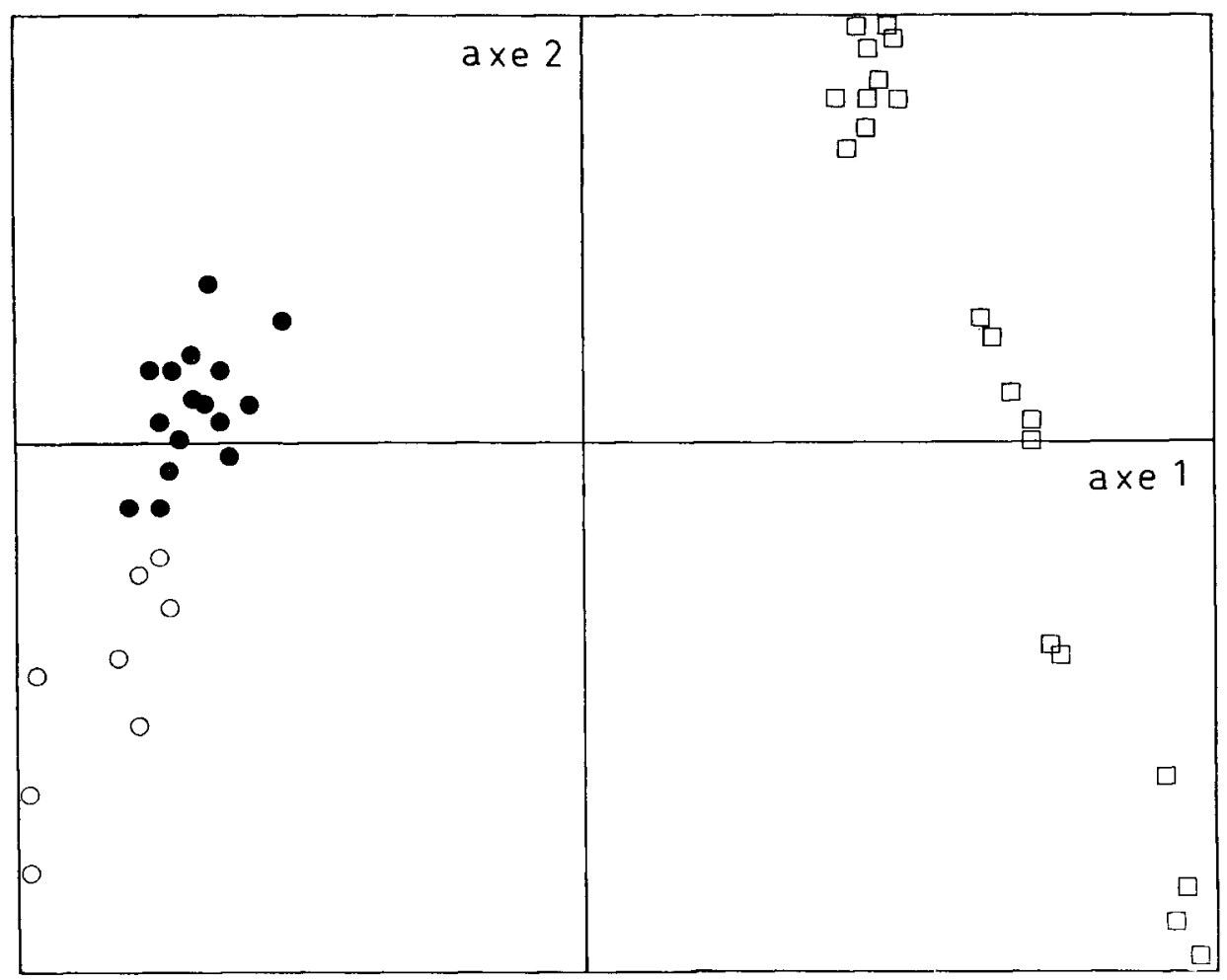

FIG. 2

Analyse factorielle des correspondances globales. Carte des relevés dans le plan des ares 1 et 2. Global reciprocal averaging. Diagram of the samples in the plane of the axes 1 and 2.

Relevé de la série mégatherme hygrophile - Sample from megathermic hygrophilic succession.

- Relevé de la série mésotherme hygrophile climacique - Sample from mesothermic hygrophilic succession-climax.

O Relevé de la série mésotherme hygrophile à Acacia heterophylla - Sumple from mesothermic hygrophilic succession-Acacia heterophylla facies. 
Nous donnons dans les tableaux 1 et 2 les listes d'espèces différenciant chacune des deux séries par rapport à l'autre. Ces listes ont été établies à partir du graphe des espèces de l'analyse factorielle, trop dense pour être reproduit ici. Elles sont assez fournies pour permettre sans équivoque l'identification de tout relevé de végétation comme appartenant à l'une ou l'autre de ces deux séries.

Les relevés s'étagent entre 0 et $800 \mathrm{~m}$ (jusqu'à $1000 \mathrm{~m}$ dans lat région sous le vent) pour la série mégatherme hygrophile et entre 1200 et $1750 \mathrm{~m}$ pour la série

\section{TABLeaU 1}

Espèces différentielles de la série mégatherme hygrophile par rapport à la série mésotherme hygrophile.

Differential species of megathermic hygrophilic succession compared with mesothermic hygrophilic succession.

Strate arborescente et arbustive :

Antidesma madagascariense

Cordemoya integrifolia

Homalium paniculatum

Psiloxylum mauritianum

Casearia coriacea

Mimusops maxima

Labourdonnaisia callophylloides

Linociera brooneana

Agauria salicifolia

Ficus lacens

Ficus mataritiana

Diospyros melanida

Coffea mataritiana

Mussisenda landia

Bertiera borbonica

Memecylon confusum

Tabaernaemontana maturitiana

Eugenia cymosa

Tambourissat quadrifida

Calophyllam tacamahaca

Polyscias repanda

Xylopia richardii

Dombeya ciliata

lyophorbe indica

Pandanas parparascens

Allophyllas cobbe

Strate herbacéc:

Phaius tetragonus

Nephrolepis biserrata

Selaginella falcata

Ctenitis sp. (n"4153)
Lianes :

Danais fragans

Tylophora laevigata

Cnestis shobra

Epiphytes

Algratecum matritianum

Ansrasem cucullatum

Angratectm squamatam

Angracum ramosum

Angraecum pectinatum

Polystachia maturitiana

Bulbophyllum conicum

Bulbophyllum variegatum

Bulbophyllam sp. (n" 3273)

Bulbophyllum sp. (n" 3444)

Oberomia equitans

Elaphoglossum tomentosum

Angraecopsis parviflora

Asplenium affine

Phymatodes scolopendria

Trichomanes bipunctatum

Trichomanes caspidatum

Nephrolepis biserrata

Ophioglossam pendulam

Hymenophyllam fumarioides

Pifer pyrifolitam

Peperomia baroni

Belvisia spicata

Procris pedanculata

Lomariopsis pollicina 


\section{TABleav 2 \\ Espèces différentielles de la série mésotherme hygrophile par rapport à la série mégatherme hygrophile.}

Differential species of mesothermic hygrophilic succession compared with megathermic hygrophilic succession.

Strate arborescente et arbustive :

Dombeya punctata

Dombeya reclinata

Dombeya pilosa

Monimia rotundifolia

Tambourissa elliptica

Evodia obtusifolia var. arborea

Hypericum lanceolatum

Eugenia cotinifolia

Claorylon glandulosum

Psychotria gaertneroides

Bertiera rufa

Psiadia laurifolia

Cyathea glauca

En abondance-dominance :

Weinmamia tinctoria

Strate herbacée :

Isachne maturitiana

Pilea rupipendia

Thelypteris heteroptera

Astelia hemichrysa
Epiphytes :

Peperomia boivini

Astelia hemichrysa

Bulbophyllum nutans

Jumellea triquetra

Angraecum multiflorum

Elaphoglossum hybridum

Elaphoglossum splendens

Elaphoglossum falcatum

Elaphoglossum auberiii

Asplenium petiolulatum

Asplenium aethiopicum

Polystichum ammifolium

Dryopteris aquilinoides

Vittaria isoetifolia

Pleopeltis excavata

Blotiella pubescens

Ctenitis sp. ( $\mathrm{n}$ "4019)

mésotherme hygrophile. Entre 800 et $1200 \mathrm{~m}$, on rencontre le plus souvent une formation non forestière, les fourrés très hygrophiles à Pandanus montanus qui se rattache à la série mésotherme.

11 faut noter d'autre part sur la figure 2 que les relevés de la forêt de tamarins des hauts ne se séparent pas de ceux du reste de la série mésotherme hygrophile. Là aussi, il est donc confirmé que ce type de forêts doit bien être envisagé comme une sous-unité (à caractère dynamique selon CADET) de la série mésotherme.

Par contre, on observe un étirement le long de l'axe 2, aussi bien des relevés de la série mésotherme que de ceux de la série mégatherme. Pour ces derniers, il s’agit d'un gradient altitudinal qu'une analyse partielle va nous permettre de préciser.

\subsection{Analyse partielle des relevés de la série mégatherme hygrophile}

L'axe 1 de l'analyse représente 22 p. 100 de la variance totale, ce qui montre là encore la présence d'un facteur fortement explicatif. Les relevés sont, par contre, répartis de façon relativement homogène au long de cet axe (fig. 3). 


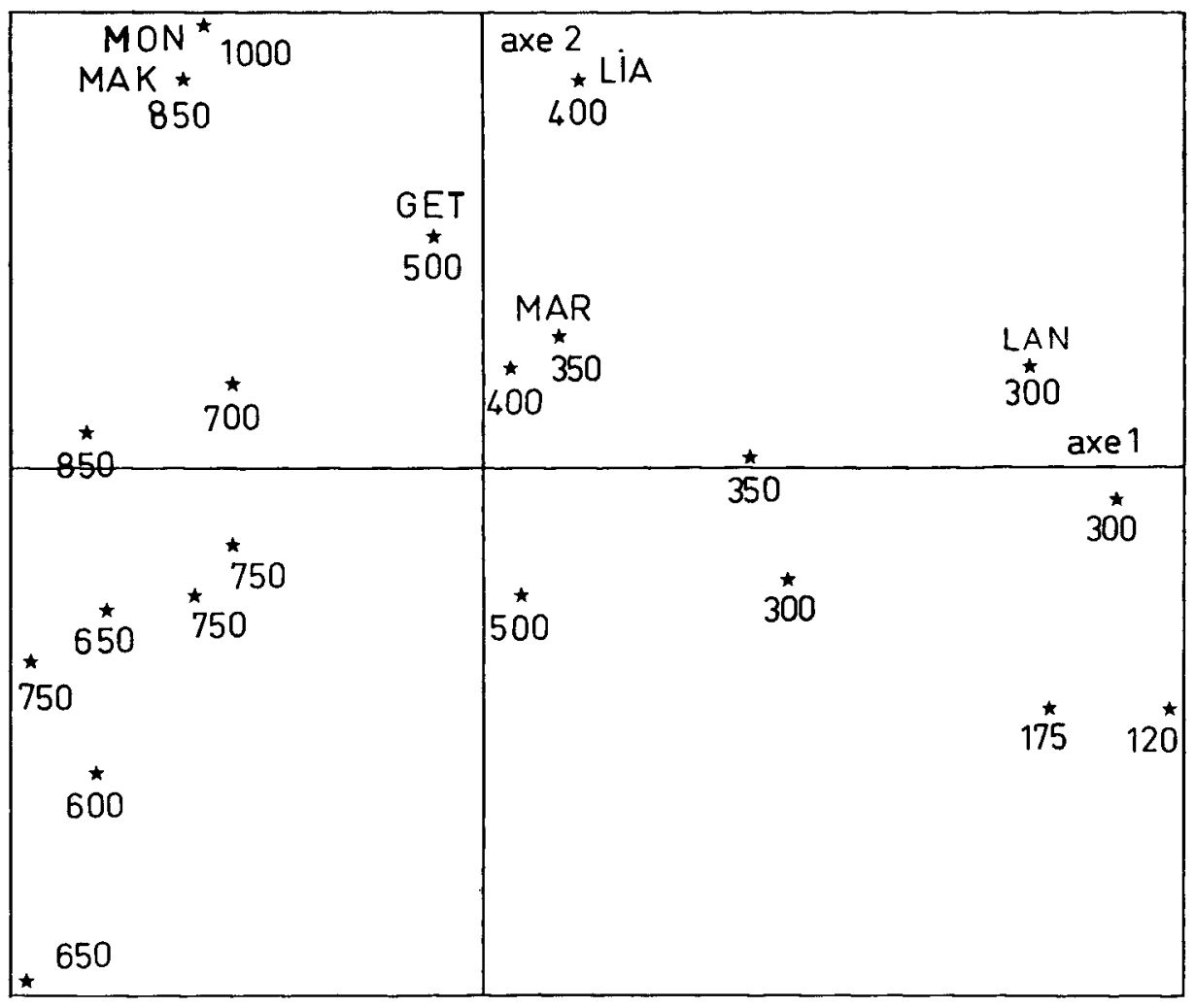

FIG. 3

Analyse factorielle des correspondances partielles des relevés de la série mégatherme hygrophile.

Partial reciprocal averaging of megathermic hygrophilic forest samples.

Carte des relevés dans le plan des axes 1 et 2 . Les altitudes sont portées sous chaque relevé. Pour certains, la localisation exacte est indiquée - Diagram of the samples in the plane of the axes $I$ and 2. Altitudes are marked under each sample. In addition, the exact locality is mentionned for some ones:

MON : Massif de la montagne de Saint-Denis.

Massif of the mountain of Saint-Denis.

MAK : Plaine des Makes.

Plain of Makes.

LIA : Plaine des Lianes.

Plain of Lianes.

GET : Grand-Etang.

Grand Etang.

MAR : Rivière des Marsouins.

$$
\text { River of Marsouins. }
$$

LAN : Vallée de la rivière Langevin. Vale of the river Langevin. 
Les altitudes de chaque rclevé, portées sur le graphique, montrent clairement que ce facteur de variation de la composition floristique des relevés est lić au gradient altitudinal : sur les abcisses positives sont situés les relevés d'altitude inférieure à $500 \mathrm{~m}$, opposés aux relevés d’altitude supérieure à $600 \mathrm{~m}$ sur les abcisses négatives. Il ne peut s'agir ici que du facteur thermique dont les variations avec l'altitude sont évidentes en raison du relief accusé de l'île.

\section{TABLEAU 3}

Espèces différentielles de la forêt mégatherme hygrophile de basse altitude par rapport à la forêt mégatherme hygrophile de moyenne altitude.

Differential species of low altitude megathermic hygrophilic forest compared with middle altitude megathermic hygrophilic forest.

Strate arborescente et arbustive :

Erythroxylon laurifolium *:

Sideroxylon borbonicum var. capuroni *:

Diospyros melanida *:

Ficus morifolia *:

Ficus rubra*

Hyophorbe indica*:

Acantophoenix rubra *

Agauria salicifolia*

Allophyllus cobbe *

Memecylon confusum *

Tabernaemontana maritiana*

Myonimia myrtifolia:

Bertiera borbonica*

$X y l o p i a$ richardii *

Eugenia paniculata*

Leea sambucina *

Mimusops maxima $* *$

En abondance-dominance :

Labourdonnaisia callophylloides:

Psychotria boryana

Lianes :

Cnestis glabra*
Strate herbacéc :

Nephrolepis biserrata *:*

Phymatodes scolopendria ***

Phaitus tetragonus **

Selerginella sp. (n"1195)*

Begonia aptera **

En abondance-dominance :

Asplenium lineatum

Ctenitis sp. (n"4153)

Epiphytes:

Vittaria zoosterifolia *

Trichomanes bipunctatum *:

Trichomanes cuspidatum *:

Ophioglossum pendulum **

Plymatodes scolopendria $* *: *$

Nephrolepis biserrata *:*:

Aspleniam pellucidum **

Asplenium nidus *

Lomariopsis pollicina $* *$

Bulbophyllum variegatum :

Aeranthes arachnites **

Bullophyllum sp. (n" 3273) **

Polystachia maturitiana $*$ *

Ansratecum mauritianum *

Angraecum shurneum *

Angraecum pectinatum *

Procris pedunculata*

Arthropteris boutoniana *

Seuil de signification de la différence des fréquences de présence de chaque espèce dans les deux types de forêt - Level of signification of the difference between the presences for each species in the two types:

\footnotetext{
$\because \quad 10$ p. 100

I p. 100

1 p. 1000 .
} 
De très nombreuses espèces sont liées à ce gradient. Les tableaux 3 et 4 donnent la liste des espèces différenciant chacune des deux zones d'altitude. Plusieurs remarques s’en déduisent :

- La forêt mégatherme de basse altitude est plus riche en espèces différentielles, et celles-ci sont de meilleures indicatrices que pour la forêt mégatherme de moyenne altitude.

- Les espèces différentielles de la forêt mégatherme de moyenne altitude par rapport à la forêt mégatherme de basse altitude sont souvent des espèces que l'on retrouve aussi dans la zone des forêts mésothermes. Tel est le cas de Phyllanthus phyllirifoliam, Evodia borbonica, Dombeya punctata, Carex walhenbergiana, Calanthe sylvatica, Jumellea triquetra...

\section{TABLI:AU 4}

Espèces différentielles de la forèt mégatherme hygrophile de moyenne altitude' par rapport à la forèt mégatherme hygrophile de basse altitude.

Differential species of middle altitude megathermic hygrophilic forest compared with low altitude megathermic hygrophilic forest.

Strate arborescente et arbustive :

Pltyllanthus phyllirifolium **:*

Evodia borbonica *

Evodia borbonica var. acuminata *

Dombeya punctata *

Badula grammisticta :

Badula borbonica

Vernonia fimbrillifera *

Faujasia flexuosa

Quivisia ovata

Pandanus montanus

Eugonia platyphylla

Maillardia borbonica

Mussaenda landia

Dracaena reflexa :

Weimmannia tinctoria"

Geniostoma borbonicum *

En abondance-dominance :

Cordemoya integrifolia

Pandanus purpurascens
Strate herbacée :

Carex walhenbergiana *

Calanthe sylvatica*

Habenaria sigillum

Habenaria praealta

Pteris woodwardioides *

En abondance-dominance :

Selaginella falcata

Lianes :

Clematis matitiana

Senecio penicillatus

Epiphytes :

Jumellea triquetra*

Angraecum squamatum *

Peperomia baroni *

Beclardia macrostachya

Polystachia fusiformis *

Elaphoglossum richardii *

Mais il existe manilestement un noyau d'espèces cantonnées à la seule forêt de moyenne altitude et qui en sont done de véritables caractéristiques. Ce sont : Badala grammisticta, Vernonia fimbrillifera, Evodia borbonica var acuminata, Peperomia baroni, Angraccum squamatum, Polystachia fusiformis, Elaphoglossum richardii, Pteris woodwardioides et, à un moindre degré : Quivisia ovata, Pandanus montamus, Eugenia platyphylla, Maillardia borbonica, Mussaenda landia, Faujasia flexuosa, Habenaria sigillam et Habenaria praealta. Trois espèces sont physionomiquement représentatives par leur abondance-dominance nettement plus forte en forêt de moyenne altitude : Cordemoya integrifolia, Pandanus purparascens et Selaginella falcata. 
On retrouve dans cette liste despèces une partie de celles citées par Rıvals comme caractéristique de la forêt complexe de moyenne altitude.

Le deuxième axe de l'analyse factorielle ne représente plus que 9 p. 100 de la variance totale, mais il a une signification importante. II oppose d'une part, sur les ordonnées positives, les seuls relevés de la zone sous le vent (massif de la montagne de Saint-Denis et plaine des Makes) ou en limite (vallée de la rivière Langevin), ainsi que les relevés effectués au Nord de Sainte-Anne sur les lithosols des remparts encaissant les vallées (Grand-Etang, rivière des Marsouins, plaine des Lianes) et, d'autre part, sur les ordonnées négatives, les relevés de la zone Sud-Est de l’île, entre SainteAnne et la rivière Langevin. It traduit done une différenciation géographique de la série mégatherme hygrophile, liće très certainement à la pluviométrie mais aussi à la topographie : sur les remparts abrupts, le drainage latéral est tel que l'on retrouve des conditions de sécheresse relative équivalentes à celles de la zone sous le vent. Il est difficile de mettre en évidence des espèces liées à ce gradient vu le peu de relevés dont on dispose en zone sous le vent. Tout au plus peut-on noter, dans cette zone, la présence de Badula borbonica (sans doute sagit-il dans ce cas de la variété borbonica), Acalypha colorata. Clasxylon parviflormm. Dombeya elegans et Olea lancea.

\section{Conclusion}

Deux conclusions se dégagent de cette étude :

- Sur le plan phytosociolugique, si la séparation introduite par Cadet (1980) entre série mégatherme hygrophile et série mésotherme hygrophile est parfaitement justifiće, il semble par contre qu'il soit nécessaire de conserver, comme le lait Rivals (1952) une subdivision majeure au sein de la série mégatherme hygrophile entre forêts de basse altitude et forêts de moyenne altitude, la limite se situant vers $500 \mathrm{~m}$. Nos analyses factorielles ont en effet montré l'existence d'une forte variabilité floristique lice à l'altitude. Nous proposons une division en deux sous-ensembles afin de disposer d'unités opérationnelles de gestion, tout en ayant conscience que le passage de l'un à l'autre est progressil et marqué par des espèces «à cheval sur cette limite, tel le bois maigre (Nuxia verticillata). le losto cafe (Gaertnera vaginata). le sabre (Machaerina iridifolia)... Derrière le facteur thermique apparaît une différenciation géographique, quiil faudrait pouvoir préciser sur la base d'un nombre de relevés plus important.

D’un point de vue plus général, le schéma phytosociologique proposé actuellement pour la Réunion doit pouvoir encore être largement affiné. Les unités climaciques définies sont en effet très hétérogènes. Or, la base d'une gestion forestière efficace est un découpage le plus fin possible des zones à aménager en unités écologiquement homogènes (stations). Ce résultat est loin d'être atteint à la Réunion.

- Dun point de vue forestier, la mise en évidence d'une unité mégatherme de moyenne altitude, avec un cortège floristique lié, devrait être prise en compte lors des interventions sylvicoles, en particulier pour le choix des essences indigènes ou exotiques à cultiver, dans la commune de Saint-Philippe et la partie Sud de la commune de SainteRose (Bois blanc). 
Les forestiers avaient d'ailleurs déjà identifié la zone des forêts mégathermes hygrophiles de basse altitude, qu'ils ont baptisée "forêt de bois de couleur des bas ». comme celle où la régénération naturelle assistée des espèces autochtones est relativement aisée. Les réalisations conduites au cours des cinq dernières années ont démontré que la zone des forêts mégathermes hygrophiles de moyenne altitude était susceptible d'enrichissement par plantations, en particulier d'une espèce exotique particulièrement bien adaptée à cette plage d'altitude, le camphrier (Cinnamomum camphora).

Nous confirmons enfin le bien fondé, dans l'aménagement de la forêt de SaintPhilippe, de l'extension en altitude de la réserve de Mare-Longue, englobant ainsi les zones originales comprises entre 500 et $800 \mathrm{~m}$.

Recule le 22 mars 1985.
Accepté le 14 mai 1985.

\section{Remerciements}

Nous tenons à exprimer nos remerciements au Centre d'Etudes de Recherches et de Formation de la Réunion où ont pu être menées à bien les analyses informatiques.

\section{Summary \\ Typology of Reunion island woodlands}

We analyse the vegetational samples of Reunion island forest published in CaDET (1980). Data processing using reciprocal averaging allow us to specify the subdivisions of Reunion forest. The validity of the gap between hygrophilic megathermic succession and hygrophilic mesothermic succession is confirmed. On the other hand, it appears two subunits of the megathermic succession : the low altitude forest and the middle altitude forest. Characteristic species of each one of these units are identified. Further, the megathermic succession secms to be also differentiated along a geographical gradient which separates the South East of the island, exposed to the wind from the North and West, protected from the wind.

Sylvicultural implications are discussed, particulary the need for an active conservation program. In effect, the goal of the local Forest Service is to substitute the great initial variability for only one or two species.

\section{Références bibliographiques}

CadeT T., 1980. La végétation de lîle de la Réunion. Etude phytoécologique et phytosociologique. Cazal, Saint-Denis de la Réunion, 312 p.

Dagnelie P., 1960. - Contribution à l'étude des communautés végétales par l'analyse factorielle. Bull. Service Carte phytogéographique, 5 (1-2), 1-195.

LacosT: A., Roux M., 1971 et 1972. L'analyse multidimensionnelle en phytosociologie et en écologie. Application à des données de l'étage subalpin des Alpes-Maritimes. I. L'analyse des données floristiques. II. L'analyse des données écologiques et lanalyse globale. Ecol. Plant., 6, 353-369 et 7, 125-146.

Miguex J.M., 1955. Boisement et régénération de forêts reliques en zone tropicale humide. Les forêts de Saint-Philippe à la Réunion. Rev. For. Fr., 6, 187-200.

Rivass P., 1952. Etudes sur la végétation naturelle de l'île de la Réunion. Douladoure, Toulouse, $214 \mathrm{p}$. 